\title{
Einstellung von hausärztlichen Patientinnen und Patienten zu schul- und komplementärmedizinischen Verfahren - ein Stimmungsbild
}

\author{
Silke Henzen ${ }^{\mathrm{a}}$ Patrick Scheiwiler ${ }^{\mathrm{b}}$ Vladimir Sibalic ${ }^{\mathrm{c}}$ \\ a'Landarztpraxis, Sevelen, Schweiz; \\ ${ }^{b}$ Arnegg, Schweiz; \\ 'St. Gallen, Schweiz
}

\section{Schlüsselwörter}

Komplementärmedizin · Phytotherapie · Homöopathie · Herzinfarkt · Funktionelle Magen-Darm-Störungen · Depression

\section{Zusammenfassung}

Hintergrund: Komplementärmedizinische Medikamente erfreuen sich gemäss Literaturangaben einer grossen Beliebtheit im freien Arzneimittelmarkt. Eine Analyse der Patientenmeinungen in schulmedizinischen Praxen bezüglich des Einsatzes von Phytotherapie und Homöopathie bei der Behandlung von häufigen somatischen Erkrankungen unterschiedlichen Schweregrades sowie der Depression fehlt. Methoden: 203 Patienten (108 Frauen, 95 Männer) in fünf Ostschweizer Allgemeinmedizinpraxen gaben auf einer Skala von 1 bis 10 eine persönliche Einschätzung zur Wirksamkeit und zum Nebenwirkungspotenzial von Medikamenten aus der Schulmedizin, der Phytotherapie und der Homöopathie ab. Zudem wurde gefragt, welcher Therapieform der Vorzug gegeben wird. Die zu behandelnden Erkrankungen waren Herzinfarkt, funktionelle Magen-Darm-Störungen und Depression. Die Resultate wurden nach Geschlecht, Alter, Begleiterkrankungen und Schulbildung ausgewertet. Ergebnisse: 74\% der Befragten erwarten, dass in der hausärztlichen Sprechstunde auch komplementärmedizinische Methoden angeboten werden. Die Schulmedizin wird bei Herzinfarkt und Depression favorisiert. Die Phytotherapie oder eine Kombination wird zur Behandlung des unruhigen Magen-Darm-Trakts bevorzugt. Frauen und unter 40Jährige bevorzugen eher die Komplementärmedizin. Die Wirksamkeit und Nebenwirkungsrate der Phytotherapie und Homöopathie wird gleichgesetzt. Die Schulbildung beeinflusst die Beurteilung gering. Schlussfolgerung: In den letzten 10 Jahren hat sich die Patienteneinstellung kaum verändert, trotz neuer wissenschaftlicher Erkenntnisse. Die positiven Resultate der wissenschaftlichen Forschung in der Komplementärmedizin sollten an die Patienten weitergegeben werden. Dies ist eine Aufgabe der Ärztinnen und Ärzte.

(c) 2016 S. Karger GmbH, Freiburg

\section{Keywords}

Complementary medicine $\cdot$ Phytotherapy $\cdot$ Homeopathy . Myocardial infarction · Irritable bowel syndrome · Depression

\section{Summary}

Primary Care Patients' Attitude to Conventional and Complementary Medicine: A Presentation of People's Feelings and Preferences

Background: As described in the literature, complementary medicine is very popular. When visiting a general practice in Switzerland, however, the preferences of the patients regarding the use of phytotherapy and homeopathy for the treatment of depression and somatic illnesses are still unknown. Methods: 203 patients (108 women, 95 men) in five general practices in eastern Switzerland were requested to specify the effectiveness and the side effects of conventional medicine, phytotherapy, and homeopathy on a scale from 1 to 10. The patients' preferred type of therapy was also asked about. The diseases treated were myocardial infarction, irritable bowel syndrome, and depression. The results were evaluated according to sex, age, comorbidities, and education. Results: $74 \%$ of the patients expect complementary medicine to be offered in general practices. Conventional medicine is favored in the case of myocardial infarction and depression. Phytotherapy is preferred in the treatment of irritable bowel syndrome. Women and patients $<40$ years prefer complementary medicine. The effectiveness and side effects of phytotherapy and homeopathy are classified equally. Education only had little influence on the assessment. Conclusion: Over the past 10 years, the attitude of the patients has not changed, despite new scientific evidence. The positive results of scientific research in complementary medicine should be passed on to the patients. This is a responsibility of the doctors.

\section{KARGER \\ Fax +497614520714}

Dr. med. Silke Henzen

Landarztpraxis

Velturrietstrasse 5,9475 Sevelen, Schweiz

silke.henzen@landarztpraxis.ch 


\section{Einführung}

Die Anwendung von komplementärmedizinischen Arzneimitteln ist weit verbreitet. In den USA wurden im Jahr 2007 gemäss einer Studie von Nahin et al. [1] 33,9 Milliarden USD für Komplementärmedizin ausgegeben. Davon wurden zwei Drittel für komplementärmedizinische Produkte und ein Drittel für Konsultationen eingesetzt [1]. Bei einer Umfrage in Deutschland im Jahr 2010 gaben $70 \%$ der erwachsenen Bevölkerung an, bereits einmal Naturheilmittel verwendet zu haben. Unter den Nichtnutzern könnten sich 7\% vorstellen, im Krankheitsfall darauf zurückzugreifen; weitere $12 \%$ würden es vom konkreten Fall abhängig machen. Nur 7\% lehnten Naturheilmittel ab [2]. Das Schweizer Volk hat sich mit der Abstimmung vom 17. Mai 2009 für eine Integrierung der Komplementärmedizin in das Gesundheitssystem entschieden, indem es für die Aufnahme der Komplementärmedizin in die Grundversicherung gestimmt hat. In unserem Nachbarland Liechtenstein lässt sich die Bevölkerung gemäss einer aktuellen Umfrage am liebsten schulmedizinisch behandeln. Nur 12,7\% der Bevölkerung haben eine homöopathische Behandlung in Anspruch genommen, und nur 5\% haben sich auf eine phytotherapeutische Behandlung eingelassen [3]. In einer Studie des Bundesamts für Gesundheit (BAG) gaben lediglich 10,6\% der in der Schweiz befragten Personen an, komplementärmedizinische Massnahmen (Homöopathie, Phytotherapie, Traditionelle Chinesische Medizin (TCM), Antroposophische Medizin, Neuraltherapie) in Anspruch zu nehmen. An erster Stelle stand dabei die Homöopathie und an zweiter Stelle die Phytotherapie. Dabei wurde die Homöopathie häufig von einem Arzt durchgeführt, während die Phytotherapie häufiger von Therapeuten angeboten wurde [4]. Wie gross der Wunsch nach einer komplementärmedizinischen Behandlung in einer schulmedizinisch orientierten Arztpraxis ist, ist nicht bekannt. Auch ist nicht bekannt, ob spezielle Patientengruppen eher eine komplementärmedizinische Behandlung beanspruchen als andere.

Das Ziel der vorliegenden Studie war, die aktuelle Einstellung der Patienten zur Komplementärmedizin in schulmedizinischen Praxen in der Ostschweiz zu erfassen. Hierfür wurden drei unterschiedliche Krankheitsbilder gewählt:

- Ein Herzinfarkt, der als lebensbedrohlich gilt. Die Risikofaktoren lassen sich jedoch gut medikamentös behandeln, im Sinne einer Primär- oder auch Sekundärprophylaxe.

- Ein unruhiger Magen-Darm-Trakt als eine die Lebensqualität beeinträchtigende, aber harmlose Erkrankung.

- Eine Depression als die Lebensqualität beeinträchtigende, aber potenziell auch lebensbedrohliche Erkrankung.
Es wurden bewusst drei häufige, allgemein gehaltene Krankheitsbilder gewählt, die den Probanden bekannt sind.

Bei der Studie interessierten die geschlechterspezifische und die altersabhängige Verteilung. Des Weiteren waren die Auswirkung der Schulbildung auf die Einnahme von schulmedizinischen, phytotherapeutischen und homöopathischen Arzneimitteln oder einer Kombination dieser Arzneimittel durch Patienten, die eine allgemeinmedizinische Praxis aufsuchen, von Bedeutung. Zusätzlich wurde die Einstellung zu diesen Arzneimitteln bei Patienten untersucht, die bereits an einer der oben genannten Erkrankungen leiden.

\section{Patienten und Methoden}

\section{Einschlusskriterien}

An der Befragung beteiligten sich fünf Ärzte, davon drei aus dem Kanton St. Gallen, einer aus dem Kanton Thurgau und einer aus dem Kanton Zürich. Im Rahmen des «Kollegiums für forschende Hausärzte» werden in diesen hauptsächlich schulmedizinisch geführten Praxen regelmässig Hausarztstudien durchgeführt. Die Befragung umfasste den Zeitraum vom 24.03.2014 bis zum 10.04.2014. Alle Patienten mit guten Deutschkenntnissen, die älter als 18 Jahre waren und die in diesem Zeitraum zu einer Konsultation in die Praxis kamen, wurden gefragt, ob sie an dieser Studie teilnehmen möchten. Sie erhielten einen Fragebogen mit folgender Erklärung über die Herstellungsmethoden schulmedizinischer, phytotherapeutischer und homöopathischer Arzneimittel:

«Schulmedizinische Medikamente werden oft synthetisch hergestellt. Pflanzliche Medikamente werden aus Pflanzen gewonnen. Homöopathische Medikamente enthalten sehr stark, mehrere tausendfach verdünnte Wirkstoffe.»

Anschliessend unterschrieben sie die Einverständniserklärung für eine anonyme Auswertung. Des Weiteren wurden sie darüber informiert, dass sie die Teilnahme an der Studie jederzeit widerrufen können.

\section{Fragebogen}

Im Fragebogen wurden die Patienten gebeten, über ihr Alter, ihr Geschlecht, ihre Herkunft und ihre Schulbildung sowie über bereits vorbestehende Erkrankungen oder Medikamenteneinnahmen Auskunft zu geben. Danach wurden ihnen folgende Fragen gestellt:

- Frage 1: Wie schätzen Sie die Wirksamkeit der Medikamente gegen Herzinfarkt ein?

- Frage 2: Wie stark schätzen Sie die Nebenwirkungen der Medikamente gegen Herzinfarkt ein?

- Frage 3: Wie stark schätzen Sie die Wirksamkeit der Medikamente bei nervösen Magen-Darm-Beschwerden (Blähungen, Bauchkrämpfe, Völlegefühl) ein?

- Frage 4: Wie stark schätzen Sie die Nebenwirkungen der Medikamente gegen nervöse Magen-Darm-Beschwerden ein?

- Frage 5: Wie stark schätzen Sie die Wirksamkeit der Medikamente gegen Depression ein?

- Frage 6: Wie stark schätzen Sie die Nebenwirkungen der Medikamente gegen Depression ein?

- Frage 7: Welche Medikamente würden Sie sich gegen Herzinfarkt verschreiben lassen?

- Frage 8: Welche Medikamente würden Sie sich gegen nervöse MagenDarm-Beschwerden verschreiben lassen? 
Tab. 1. Demographie $(n=203$ Patienten $)$

\begin{tabular}{lc}
\hline & Patienten \\
\hline Durchschnittliches Alter, Jahre (\%) & $48(18-86)$ \\
\hline Geschlecht, n (\%) & $108(53)$ \\
Weiblich & $95(47)$ \\
Männlich & \\
\hline Schulbildung, n (\%) & $16(8)$ \\
Keine Lehre & $106(52)$ \\
Lehre & $60(30)$ \\
Höherer Schulabschluss bzw. Universität & $21(10)$ \\
Keine Angaben & \\
\hline Gesundheitszustand, n (\%) & $76(37)$ \\
Keine chronische Erkrankung (gesund) & $47(23)$ \\
Herz-Kreislauf-Erkrankungen (arterielle Hypertonie, & \\
$\quad$ koronare Herzerkrankung, Insult) & $20(10)$ \\
Psychische Erkrankung & $1(0,5)$ \\
Chronische Darmerkrankung & $78(38,5)$ \\
Keine Angaben &
\end{tabular}

- Frage 9: Welche Medikamente würden Sie sich gegen Depression verschreiben lassen?

Die Patienten wurden gebeten, jeweils in einem Kästchen eine Bewertung von 1 bis 10 für die verschiedenen Therapiemöglichkeiten (schulmedizinische Medikamente, pflanzliche Medikamente, homöopathische Medikamente oder eine Kombination von Medikamenten) abzugeben:

- Frage 1, 3 und 5: 1 = Fast nicht wirksam, $10=$ sehr stark wirksam.

- Frage 2, 4 und 6: 1 = Fast keine Nebenwirkungen, 10 = starke Nebenwirkungen.

- Frage 7, 8 und 9: 1 = Würde ich mir nicht verschreiben lassen, 10 = würde ich mir unbedingt verschreiben lassen.

- Abschlussfrage 1: Ich habe mir bereits Gedanken über die Wirksamkeit von schulmedizinischen, pflanzlichen und homöopathischen Medikamenten gemacht. - Ja; noch nie; manchmal.

- Abschlussfrage 2: Ich habe bereits pflanzliche/homöopathische Medikamente aus nichtärztlicher Quelle bezogen. - Ja; nein.

- Abschlussfrage 3: Sollte Ihr Hausarzt vor einer Behandlung immer mehrere Behandlungsmöglichkeiten (Schulmedizin, pflanzliche Therapie, Homöopathie) anbieten, sofern es diese gibt? - Ja; nein; weiss nicht.

\section{Auswertung}

Für die Auswertung wurden verschiedene Gruppen gebildet:

Die erste Auswertung umfasste die Daten aller Probanden; danach wurde eine Aufteilung der Daten nach Geschlecht (männlich, weiblich) und nach Schulbildung (keine Lehre, Lehrabschluss, höherer Schulabschluss inklusive Universität, keine Angaben) vorgenommen. Die Erkrankungen der Patienten wurden in Gruppen unterteilt (keine chronischen Erkrankungen, HerzKreislauf-Erkrankungen, psychische Erkrankungen, chronische Darmerkrankungen, keine Angaben).

Die Patienten wurden zudem in drei verschiedene Altersgruppen in Jahren eingeteilt: $<40=$ junge Altersgruppe; $40-60=$ mittlere Altersgruppe; $>60$ $=$ höhere Altersgruppe.

\section{Statistik}

Die Tabellen, Grafiken und statistischen Auswertungen wurden mit dem Programm SPSS erstellt. Die Patienten gaben bei der Beantwortung der Fragen ganze Zahlen auf einer Ordinalskala von 1-10 an, sodass sich die MedianWerte zur Berechnung der Studienresultate anboten. In der Folge wurden nichtparametrische Tests zur Berechnung der Signifikanzen verwendet. Das
Signifikanzniveau zur Wirksamkeit, zu Nebenwirkungen und zur bevorzugten Therapieart der jeweiligen Medikamentengruppen bei den drei Krankheitsbildern (Herzinfarkt, unruhiger Magen-Darm-Trakt, Depression) wurde bei den Subgruppen mit dem Wilcoxon-Test errechnet, da dieser zur Analyse von zwei gepaarten abhängigen Variablen verwendet wird. Der Friedman-Test wurde zur Analyse von mehreren abhängigen Variablen (Schulmedizin, Phytotherapie, Homöopathie, Kombination) verwendet. Der Mann-Whitney-U-Test wurde zur Überprüfung des Signifikanzniveaus zwischen den Angaben der Frauen und der Männer eingesetzt - als Analyse von zwei nicht gepaarten unabhängigen Variablen. Die Angaben innerhalb einer Altersgruppe wurden mit dem Friedman-Test errechnet, weil die Variablen zueinander abhängig sind. Der Kruskal-Wallis-Test analysierte die Unterschiede zwischen den Altersgruppen, die voneinander unabhängig sind. Der Zusammenhang der Schulbildung mit der Einschätzung der Wirksamkeit, der Nebenwirkungen und der bevorzugten Therapieform wurden mit dem Kruskal-Wallis-Test gezogen. Zur bildlichen Veranschaulichung der verschiedenen Krankheitsgruppen wurden Boxplots verwendet.

\section{Ergebnisse}

300 erwachsene Patienten wurden befragt, ob sie an der Studie teilnehmen möchten. 203 Patienten sagten daraufhin zu, bei der Studie mitzumachen (Tab. 1).

\section{Ergebnisse der Hauptbefragung}

\section{Herzinfarkt (Frage 1, 2 und 7)}

Die befragten Patienten bewerten die Schulmedizin in der Prävention eines Herzinfarkts als am besten wirksam, aber auch die Kombination von schulmedizinisch wirksamen Medikamenten mit Phytotherapie oder Homöopathie wird als gut eingestuft. Phytotherapie oder Homöopathie alleine eingesetzt werden tendenziell als weniger wirksam erachtet. Die Nebenwirkungsrate der schulmedizinischen Medikamente wird als mässig eingestuft. Die Nebenwirkungsrate von Phytotherapie/Homöopathie wird signifikant geringer eingestuft.

Zusammenfassend ziehen die Patienten bei Erkrankungen wie dem Herzinfarkt die Schulmedizin hochsignifikant den anderen Therapien vor. An zweiter Stelle rangiert dabei die Kombination von schulmedizinischen mit phytotherapeutischen bzw. homöopathischen Medikamenten, an dritter Stelle die Phytotherapie und an vierter Stelle die Homöopathie (Tab. 2).

\section{Magen-Darm-Beschwerden (Frage 3, 4 und 8)}

Sowohl die Schulmedizin als auch die Kombinationstherapie wird bei Magen-Darm-Beschwerden als wirksam eingestuft, wobei die Kombination verschiedener Arzneimittel tendenziell als weniger wirksam betrachtet wird. Die Homöopathie wird gegenüber der Phytotherapie nur als geringfügig weniger wirksam eingestuft. Die Nebenwirkungsrate wird bei der Schulmedizin am höchsten, bei der Kombinationstherapie geringer eingestuft. Bei der 
Tab. 2. Einschätzung der Wirksamkeit und der Nebenwirkungen der verschiedenen Therapiemöglichkeiten sowie die bevorzugte Therapieart bei den erfragten Erkrankungen: Ergebnisse der Hauptbefragung (Skala 1-10)

\begin{tabular}{|c|c|c|c|c|c|c|}
\hline \multirow{2}{*}{$\begin{array}{l}\text { Fragestellung } \\
\text { Herzinfarkt }\end{array}$} & \multirow[t]{2}{*}{ Therapieart } & \multirow[t]{2}{*}{$\mathrm{n}$} & \multirow[t]{2}{*}{$\begin{array}{l}\text { Median } \\
(25-75 \%)\end{array}$} & \multicolumn{2}{|c|}{ Signifikanz (Wilcox) } & \multirow[t]{2}{*}{$\begin{array}{l}\text { Signifikanz } \\
\text { (Friedman) }\end{array}$} \\
\hline & & & & & & \\
\hline \multirow{4}{*}{$\begin{array}{l}\text { Geschätzte } \\
\text { Wirksamkeit }\end{array}$} & Schulmedizin & 192 & $8(7-10)$ & 1 & \multirow{4}{*}{$\mathrm{p}=0,002$} & \multirow{4}{*}{$\mathrm{p}=0,001$} \\
\hline & Phytotherapie & 190 & $5(2-6)$ & \multirow{3}{*}{$\mathrm{p}=0,001$} & & \\
\hline & Homöopathie & 189 & $4(2-5)$ & & & \\
\hline & Kombination & 187 & $7(5-9)$ & & & \\
\hline \multirow{4}{*}{$\begin{array}{l}\text { Geschätzte } \\
\quad \text { Nebenwirkungen }\end{array}$} & Schulmedizin & 191 & $6(4-8)$ & \multirow{2}{*}{$\mathrm{p}=0,001$} & \multirow{4}{*}{$\mathrm{p}=0,37$} & \multirow{4}{*}{$\mathrm{p}=0,001$} \\
\hline & Phytotherapie & 189 & $2(1-3)$ & & & \\
\hline & Homöopathie & 186 & $2(1-4)$ & \multirow{2}{*}{$\mathrm{p}=0,001$} & & \\
\hline & Kombination & 183 & $5(3-5)$ & & & \\
\hline \multirow{4}{*}{$\begin{array}{l}\text { Bevorzugte } \\
\text { Therapieart }\end{array}$} & Schulmedizin & 192 & $9(6-10)$ & \multirow{2}{*}{$\mathrm{p}=0,001$} & \multirow{4}{*}{$\mathrm{p}=0,001$} & \multirow{4}{*}{$\mathrm{p}=0,001$} \\
\hline & Phytotherapie & 186 & $5(2-8)$ & & & \\
\hline & Homöopathie & 184 & $5(1-7)$ & \multirow{2}{*}{$\mathrm{p}=0,001$} & & \\
\hline & Kombination & 182 & $6,5(4-8)$ & & & \\
\hline \multicolumn{7}{|c|}{ Magen-Darm-Beschwerden } \\
\hline \multirow{4}{*}{$\begin{array}{l}\text { Geschätzte } \\
\text { Wirksamkeit }\end{array}$} & Schulmedizin & 192 & $8(5-9)$ & \multirow[b]{2}{*}{$\mathrm{p}=0,001$} & \multirow{4}{*}{$\mathrm{p}=0,001$} & \multirow{4}{*}{$\mathrm{p}=0,001$} \\
\hline & Phytotherapie & 190 & $6(4,75-8)$ & & & \\
\hline & Homöopathie & 190 & $5(3-8)$ & \multirow[b]{2}{*}{$\mathrm{p}=0,001$} & & \\
\hline & Kombination & 181 & $7(5-9)$ & & & \\
\hline Geschätzte & Schulmedizin & 192 & $6(4-8)$ & & & \\
\hline Nebenwirkungen & Phytotherapie & 191 & $2(1-4)$ & $\mathrm{p}=0,001$ & & \\
\hline & Homöopathie & 190 & $2(1-3)$ & & $p=0,38$ & $\mathrm{p}=0,001$ \\
\hline & Kombination & 189 & $4(2-5,5)$ & $\mathrm{p}=0,001$ & & \\
\hline Bevorzugte & Schulmedizin & 190 & $6(4-8)$ & & & \\
\hline Therapieart & Phytotherapie & 188 & $7(5-9)$ & $p=0,06$ & & \\
\hline & Homöopathie & 188 & $6,5(3-7)$ & & $\mathrm{p}=0,001$ & $\mathrm{p}=0,001$ \\
\hline & Kombination & 185 & $7(5-7)$ & $\mathrm{p}=0,18$ & & \\
\hline Depression & & & & & & \\
\hline Geschätzte & Schulmedizin & 194 & $8(5-9,25)$ & $\mathrm{n}=0.001$ & & \\
\hline Wirksamkeit & Phytotherapie & 191 & $5(3-7)$ & & $\mathrm{n}=0.62$ & $\mathrm{n}=0.001$ \\
\hline & Homöopathie & 189 & $5(2,5-6)$ & $\mathfrak{p}=0.001$ & $\mathrm{p}=0,62$ & $\mathrm{p}=0,001$ \\
\hline & Kombination & 186 & $7(5-8)$ & & & \\
\hline Geschätzte & Schulmedizin & 191 & $8(5-8)$ & $\mathrm{n}=0.001$ & & \\
\hline Nebenwirkungen & Phytotherapie & 189 & $2(1-4)$ & $\mathrm{p}=0,001$ & $n=022$ & \\
\hline & Homöopathie & 187 & $2(1-4)$ & $n-0001$ & & $\mathrm{p}=0,001$ \\
\hline & Kombination & 183 & $5(3-6)$ & $\mathrm{p}=0,001$ & & \\
\hline Bevorzugte & Schulmedizin & 192 & $7(4-10)$ & $p=0,08$ & & \\
\hline Therapieart & Phytotherapie & 190 & $6(4-8)$ & & $p=0,024$ & $\mathrm{p}=0,001$ \\
\hline & Homöopathie & 187 & $5(2-8)$ & $\mathfrak{p}=0.002$ & $p-0,0 \angle 4$ & \\
\hline & Kombination & 187 & $6(4-8)$ & $\mathrm{p}=0,002$ & & \\
\hline
\end{tabular}

Phytotherapie bzw. Homöopathie werden signifikant nur wenige Nebenwirkungen erwartet.

Zusammenfassend bevorzugen die Patienten die Phytotherapie und eine kombinierte Medikation zur Behandlung von Magen-Darm-Erkrankungen (Tab. 2).

\section{Depression (Frage 5, 6 und 9)}

Die Wirkung der schulmedizinischen Medikamente allein oder eine Kombinationstherapie wird bei der psychischen Erkrankung als signifikant besser eingestuft als die Wirksamkeit von pflanzlichen oder homöopathischen Medikamenten allein. Die Nebenwirkungsrate wird bei den schulmedizinischen Medikamenten im Vergleich zur Phytotherapie als signifikant höher eingestuft, während von der Kombination bereits weniger Nebenwirkungen erwartet werden.

Tendenziell bevorzugen Patienten schulmedizinische Medikamente zur Behandlung der Depression. An zweiter Stelle folgen die pflanzlichen Medikamente und die Kombination. Die Homöopathie ist weniger beliebt als die anderen drei Therapiearten (Tab. 2).

\section{Geschlechterspezifische Ergebnisse}

\section{Herzinfarkt (Frage 1, 2 und 7)}

Bei einem Herzinfarkt setzen Frauen und Männer tendenziell auf die Schulmedizin. Es gibt keine signifikanten Unterschiede. 
Tab. 3. Bevorzugte Therapie bei Magen-DarmBeschwerden und Depression, untersucht nach Geschlecht. Fragestellung: «Welche Medikamente würden Sie sich verschreiben lassen?» ( 1 = würde ich mir nicht verschreiben lassen; $10=$ würde ich mir unbedingt verschreiben lassen)

Abb. 1. Bevorzugte Therapieart bei Herzinfarkt, untersucht nach Gesundheitszustand.

\begin{tabular}{|c|c|c|c|c|c|}
\hline Therapieart & Erkrankung & Geschlecht & $\mathrm{n}$ & Median & Signifikanz \\
\hline \multirow[t]{4}{*}{ Schulmedizin } & \multirow[t]{2}{*}{ Magen-Darm-Beschwerden } & $\mathrm{W}$ & 103 & $5(4-8)$ & \multirow{2}{*}{$\mathrm{p}=0,049$} \\
\hline & & M & 86 & $7(5-7)$ & \\
\hline & \multirow[t]{2}{*}{ Depression } & $\mathrm{W}$ & 103 & $7(4-9)$ & \multirow{2}{*}{$\mathrm{p}=0,397$} \\
\hline & & M & 88 & $7,5(4-10)$ & \\
\hline \multirow[t]{4}{*}{ Phytotherapie } & \multirow[t]{2}{*}{ Magen-Darm-Beschwerden } & $\mathrm{W}$ & 105 & $8(5-10)$ & \multirow{2}{*}{$\mathrm{p}=0,05$} \\
\hline & & M & 82 & $6(4,75-8)$ & \\
\hline & \multirow[t]{2}{*}{ Depression } & $\mathrm{W}$ & 103 & $7(5-9)$ & \multirow{2}{*}{$\mathrm{p}=0,006$} \\
\hline & & M & 86 & $5(2,75-8)$ & \\
\hline \multirow[t]{4}{*}{ Homöopathie } & \multirow[t]{2}{*}{ Magen-Darm-Beschwerden } & W & 104 & $8(5-10)$ & \multirow{2}{*}{$\mathrm{p}=0,01$} \\
\hline & & M & 83 & $5(1-8)$ & \\
\hline & \multirow[t]{2}{*}{ Depression } & $\mathrm{W}$ & 101 & $7(4-9)$ & \multirow{2}{*}{$\mathrm{p}=0,01$} \\
\hline & & M & 85 & $4(1-6)$ & \\
\hline \multirow[t]{4}{*}{ Kombination } & \multirow[t]{2}{*}{ Magen-Darm-Beschwerden } & $\mathrm{W}$ & 100 & $7(5-9)$ & \multirow{2}{*}{$\mathrm{p}=0,008$} \\
\hline & & M & 84 & $6(3-9)$ & \\
\hline & \multirow[t]{2}{*}{ Depression } & $\mathrm{W}$ & 103 & $7(5-8)$ & \multirow{2}{*}{$\mathrm{p}=0,003$} \\
\hline & & M & 83 & $5(2-8)$ & \\
\hline
\end{tabular}

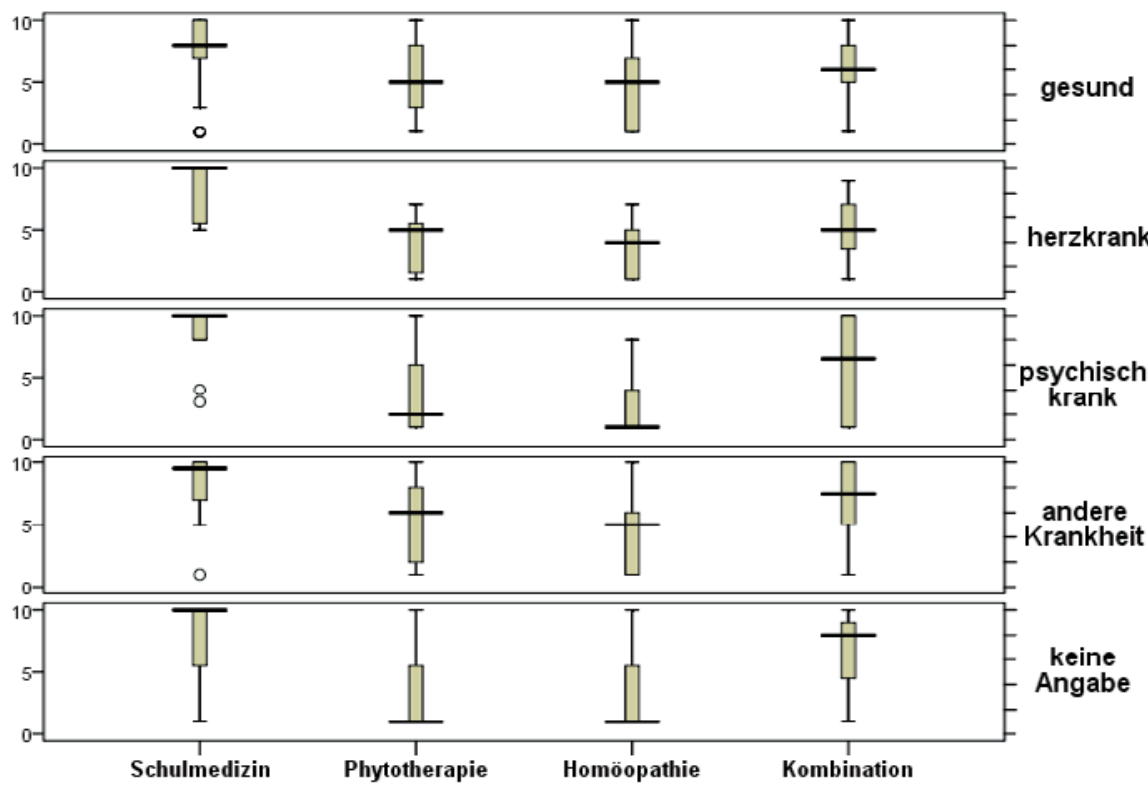

Magen-Darm-Beschwerden (Frage 3, 4 und 8)

Bei einem unruhigen Magen-Darm-Trakt setzen Männer häufiger auf die Schulmedizin als Frauen. Frauen bevorzugen die Phytotherapie signifikant. Die homöopathischen Medikamente und die Kombinationstherapie werden ebenfalls von Frauen bevorzugt (Tab. 3).

\section{Depression (Frage 5, 6 und 9)}

Bei einer Depression setzen die Männer tendenziell eher auf die Schulmedizin als Frauen. Phytotherapie, Homöopathie oder eine Kombination wird jeweils signifikant häufiger von Frauen bevorzugt (Tab. 3).

\section{Ergebnisse unter Beachtung des Gesundheitszustands der Befragten}

Da nur ein Patient an einer chronischen Darmerkrankung litt, wurde er in den Abbildungen nicht dargestellt.

\section{Herzinfarkt (Frage 1, 2 und 7)}

Alle Krankheitsgruppen ziehen gegen Herzinfarkt die Schulmedizin vor. Dahinter liegen in absteigender Reihenfolge die Kombination, die Phytotherapie und die Homöopathie (Abb. 1). 
Abb. 2. Bevorzugte Therapieart bei Magen-Darm-Beschwerden, untersucht nach Gesundheitszustand.
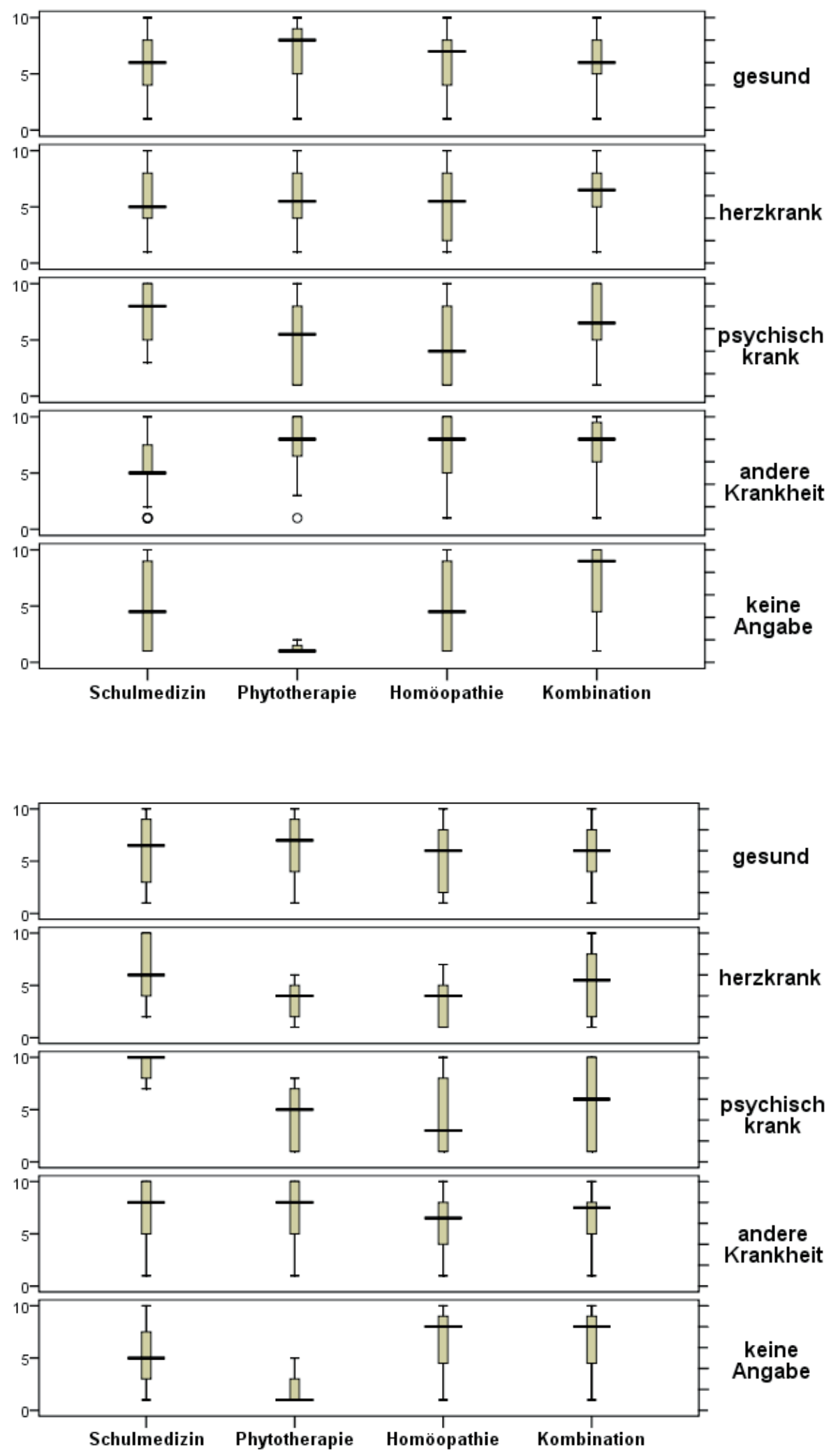

Abb. 3. Bevorzugte Therapieart bei Depression, untersucht nach Gesundheitszustand. 
Tab. 4. Bevorzugte Therapie gegen Herzinfarkt, Magen-Darm-Beschwerden und Depression, untersucht nach Altersgruppen. Fragestellung: «Welche Medikamente würden Sie sich verschreiben lassen?» ( 1 = würde ich mir nicht verschreiben lassen; $10=$ würde ich $\mathrm{mir}$ unbedingt verschreiben lassen)

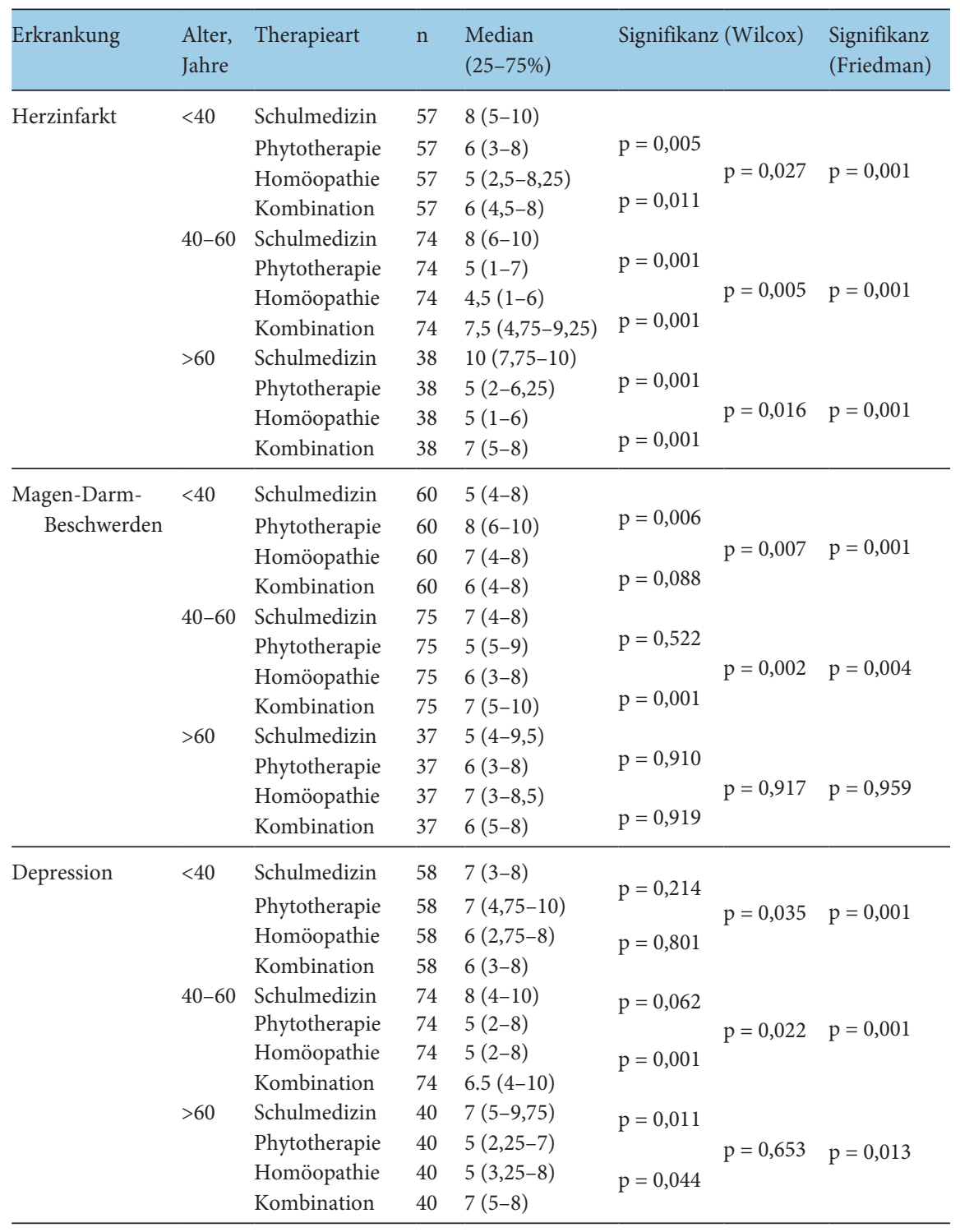

Depression (Frage 5, 6 und 9)

Bei Depressionen ziehen die Gesunden die Phytotherapie tendenziell vor. Die Herzkranken und die Gruppe der psychisch Kranken ziehen die Schulmedizin vor. Die Gruppen mit anderen Erkrankungen oder ohne Angaben unterscheiden sich in ihren Therapievorzügen nicht voneinander (Abb. 3).

\section{Altersspezifische Ergebnisse}

\section{Herzinfarkt (Frage 1, 2 und 7)}

Gegen einen Herzinfarkt möchten sich die Patienten aller Altersgruppen am liebsten mittels Schulmedizin behandeln lassen. Die Ergebnisse unterscheiden sich bei allen drei Altersgruppen bezüglich der Therapiearten signifikant voneinander. Die unter 40 -Jährigen zeigen je- doch noch eher Interesse an der Phytotherapie, während die älteren Gruppen wiederum eine Kombination bevorzugen (Tab. 4).

\section{Magen-Darm-Beschwerden (Frage 3, 4 und 8)}

Bezüglich einer Behandlung von Magen-Darm-Beschwerden finden sich in der Gruppe der unter 40-jährigen Patienten signifikante Unterschiede hinsichtlich der gewünschten Behandlung. So wird die Phytotherapie vorgezogen, während die Schulmedizin am wenigsten gern in Anspruch genommen wird. Bei den 40- bis 60-Jährigen schneidet die Homöopathie im Vergleich zu den anderen Therapien eher schlechter ab. Bei den über 60-Jährigen bestehen keine signifikanten Unterschiede (Tab. 4). 
Tab. 5. Geschätzte Wirksamkeit der Phytotherapie und Homöopathie nach Altersgruppen und Erkrankungen. Fragestellung: «Wie stark schätzen Sie die Wirksamkeit der Medikamente ein? $(1$ = fast nicht wirksam; $10=$ sehr stark wirksam)

\begin{tabular}{|c|c|c|c|c|c|}
\hline Erkrankung & Therapie & $\begin{array}{l}\text { Alters- } \\
\text { gruppen, } \\
\text { Jahre }\end{array}$ & n & $\begin{array}{l}\text { Median } \\
(25-75 \%)\end{array}$ & $\begin{array}{l}\text { Signifikanz } \\
\text { (Kruskal- } \\
\text { Wallis- } \\
\text { Test) }\end{array}$ \\
\hline \multirow[t]{6}{*}{ Herzinfarkt } & \multirow[t]{3}{*}{ Phytotherapie } & $<40$ & 58 & $5(2-5)$ & \\
\hline & & $40-60$ & 76 & $4(2-5)$ & \multirow[t]{2}{*}{$\mathrm{p}=0,17$} \\
\hline & & $>60$ & 45 & $5(2-6,5)$ & \\
\hline & \multirow[t]{3}{*}{ Homöopathie } & $<40$ & 58 & $5(2,75-6,25)$ & \\
\hline & & $40-60$ & 76 & $4(2-5)$ & \multirow[t]{2}{*}{$\mathrm{p}=0,86$} \\
\hline & & $>60$ & 44 & $3(1-5)$ & \\
\hline \multirow{6}{*}{$\begin{array}{l}\text { Magen-Darm- } \\
\text { Beschwerden }\end{array}$} & \multirow{3}{*}{ Phytotherapie } & $<40$ & 60 & $7(5-8)$ & \multirow{3}{*}{$\mathrm{p}=0,002$} \\
\hline & & $40-60$ & 78 & $5,5(4-7)$ & \\
\hline & & $>60$ & 42 & $5,5(3-7)$ & \\
\hline & \multirow[t]{3}{*}{ Homöopathie } & $<40$ & 60 & $6(4,25-8)$ & \multirow{3}{*}{$\mathrm{p}=0,072$} \\
\hline & & $40-60$ & 79 & $5(3-7)$ & \\
\hline & & $>60$ & 42 & $5(2,75-7,25)$ & \\
\hline \multirow[t]{6}{*}{ Depression } & \multirow[t]{3}{*}{ Phytotherapie } & $<40$ & 60 & $5(3,25-7,75)$ & \\
\hline & & $40-60$ & 77 & $5(3-6,5)$ & \multirow[t]{2}{*}{$\mathrm{p}=0,2$} \\
\hline & & $>60$ & 43 & $5(2-7)$ & \\
\hline & \multirow[t]{3}{*}{ Homöopathie } & $<40$ & 60 & $5(3-7)$ & \multirow{3}{*}{$\mathrm{p}=0,007$} \\
\hline & & $40-60$ & 77 & $4(2-5,5)$ & \\
\hline & & $>60$ & 41 & $4(2-6)$ & \\
\hline
\end{tabular}

\section{Depression (Frage 5, 6 und 9)}

Bei der Behandlung der Depression ziehen die 40- bis 60-Jährigen und die über 60-Jährigen die Schulmedizin vor; die Kombination folgt an zweiter Stelle. Die Unterschiede zwischen den Therapien sind signifikant. Die unter 40-Jährigen tendieren sowohl zur Schulmedizin als auch zur Phytotherapie, jedoch ohne signifikante Unterschiede (Tab. 4).

\section{Geschätzte Wirksamkeit der Phytotherapie nach Altersgruppen}

Werden die Altersgruppen untereinander verglichen, so zeigt sich nur bei den Magen-Darm-Beschwerden ein signifikanter Unterschied, nämlich bei der Altersgruppe der unter 40-Jährigen. Diese erachtet die Phytotherapie als am besten wirksam (Tab. 5).

\section{Geschätzte Wirksamkeit der Homöopathie nach Altersgruppen}

Bezüglich der Behandlung mittels Homöopathie bestehen keine signifikanten Unterschiede zwischen den Gruppen. Tendenziell zieht die Gruppe der unter 40-Jährigen die Homöopathie gegenüber den anderen Gruppen vor (Tab. 5) [5].

\section{Einfluss der Schulbildung}

Es wurden nur signifikante Unterschiede in der Beurteilung der Wirksamkeit der phytotherapeutischen oder homöopathischen Medikamente beim Herzinfarkt und bei Magen-Darm-Beschwerden gefunden.

\section{Herzinfarkt (Frage 1, 2 und 7)}

Bei der Frage zur Wirksamkeit der Medikamente gegen Herzinfarkt fand sich ein signifikanter Unterschied bei der Homöopathie und Phytotherapie. Dabei fanden die Patienten mit Lehre die Phytotherapie wirksamer als die Patienten ohne Lehre oder mit höherem Schulabschluss (Tab. 6). Bei der Frage nach Nebenwirkungen der Medikamente und der bevorzugten Therapie gegen Herzinfarkt fanden sich keine signifikanten Unterschiede.

\section{Magen-Darm-Beschwerden (Frage 3, 4 und 8)}

Patienten mit Lehre und höherem Schulabschluss fanden die Wirksamkeit der Phytotherapie besser als Patienten ohne Lehre. Patienten mit Lehre fanden die Homöopathie wirksamer (Tab. 6). Bei der Frage nach Nebenwirkungen gab es keine signifikanten Unterschiede. Bei der Frage nach den Therapievorzügen fand sich lediglich bei der Phytotherapie $(\mathrm{p}=0,02)$ ein signifikanter Unterschied. Patienten mit Lehre (Median 8) und höherem Schulabschluss (Median 7) ziehen die Phytotherapie im Vergleich zu Patienten ohne Lehre (Median $4,5)$ vor.

\section{Auswertung der Anschlussfragen}

59,8\% der Patienten haben sich schon Gedanken über die Wirksamkeit der schulmedizinischen, pflanzlichen und homöopathischen Medikamente gemacht. 23,5\% antworteten mit «manchmal» und nur 13,2\% mit «nie». Insgesamt haben sich 67,6\% der Frauen und 51,6\% der Männer Gedanken darüber gemacht $(p=0,04)$.

Die Mehrheit der Patienten (55,9\%) hat bereits pflanzliche/homöopathische Medikamente aus nichtärztlicher Quelle bezogen. Nur 11,3\% antworteten mit «manchmal» und $28,4 \%$ mit «nie». 4,4\% machten keine Angaben hier$\mathrm{zu}$.

Mehr Frauen (68,5\%) als Männer (42,1\%) haben bereits pflanzliche/homöopathische Medikamente aus nichtärztlicher Quelle bezogen $(p=0,004)$.

Die Frage, ob der Hausarzt vor einer Behandlung immer mehrere Behandlungsmöglichkeiten (schulmedizinische, pflanzliche, homöopathische) anbieten sollte, sofern es diese gibt, wurde mit einer Mehrheit von 74,5\% (Frauen $82,4 \%$; Männer 65,3\%) mit ja beantwortet. 
Tab. 6. Einschätzung der Wirksamkeit der verschiedenen Therapiemöglichkeiten abhängig von der Schulbildung. Fragestellung: «Wie stark schätzen Sie die Wirksamkeit der Medikamente ein?» (1 = fast nicht wirksam; 10 = sehr stark wirksam)

\begin{tabular}{|c|c|c|c|c|c|}
\hline Erkrankung & Therapieart & $\mathrm{n}$ & Schulabschluss & Median (25-75\%) & $\begin{array}{l}\text { Signifikanz } \\
\text { (Kruskal-Wallis-Test) }\end{array}$ \\
\hline \multirow[t]{12}{*}{ Herzinfarkt } & \multirow[t]{3}{*}{ Schulmedizin } & 18 & keine Lehre & $8(4,75-9,25-10)$ & \multirow{3}{*}{$\mathrm{p}=0,368$} \\
\hline & & 109 & Lehre & $8(7-10)$ & \\
\hline & & 62 & höherer Schulabschluss & $8(7-10)$ & \\
\hline & \multirow[t]{3}{*}{ Phytotherapie } & 18 & keine Lehre & $3,5(1,75-5)$ & \multirow{3}{*}{$\mathrm{p}=0,033$} \\
\hline & & 108 & Lehre & $5(3-6)$ & \\
\hline & & 61 & höherer Schulabschluss & $4(2-6)$ & \\
\hline & \multirow[t]{3}{*}{ Homöopathie } & 18 & keine Lehre & $3(1-5)$ & \multirow{3}{*}{$\mathrm{p}=0,006$} \\
\hline & & 107 & Lehre & $5(3-5)$ & \\
\hline & & 61 & Höherer Schulabschluss & $3(1-5)$ & \\
\hline & \multirow[t]{3}{*}{ Kombination } & 16 & keine Lehre & $7(4-8,75)$ & \multirow{3}{*}{$\mathrm{p}=0,245$} \\
\hline & & 107 & Lehre & $8(5-9)$ & \\
\hline & & 61 & höherer Schulabschluss & $7(5-8)$ & \\
\hline \multirow{12}{*}{$\begin{array}{l}\text { Nervöse Magen-Darm- } \\
\text { Beschwerden }\end{array}$} & \multirow[t]{3}{*}{ Schulmedizin } & 18 & keine Lehre & $7,5(4,75-10)$ & \multirow{3}{*}{$\mathrm{p}=0,997$} \\
\hline & & 109 & Lehre & $8(5-9)$ & \\
\hline & & 62 & höherer Schulabschluss & $8(5-9)$ & \\
\hline & \multirow[t]{3}{*}{ Phytotherapie } & 17 & keine Lehre & $3(1-5)$ & \multirow{3}{*}{$\mathrm{p}=0,002$} \\
\hline & & 109 & Lehre & $6(5-8)$ & \\
\hline & & 61 & höherer Schulabschluss & $6(5-8)$ & \\
\hline & \multirow[t]{3}{*}{ Homöopathie } & 17 & keine Lehre & $5(2-8)$ & \multirow{3}{*}{$\mathrm{p}=0,01$} \\
\hline & & 109 & Lehre & $8(5-9)$ & \\
\hline & & 62 & höherer Schulabschluss & $5,5(4-7)$ & \\
\hline & \multirow[t]{3}{*}{ Kombination } & 17 & keine Lehre & $5(2-8))$ & \multirow{3}{*}{$\mathrm{p}=0,69$} \\
\hline & & 109 & Lehre & $8(5-9)$ & \\
\hline & & 62 & höherer Schulabschluss & $7,5(5-8)$ & \\
\hline \multirow[t]{12}{*}{ Depression } & \multirow[t]{3}{*}{ Schulmedizin } & 18 & keine Lehre & $7(4,75-8,25)$ & \multirow{3}{*}{$\mathrm{p}=0,343$} \\
\hline & & 111 & Lehre & $8(5-10)$ & \\
\hline & & 62 & höherer Schulabschluss & $8(6-9)$ & \\
\hline & \multirow[t]{3}{*}{ Phytotherapie } & 18 & keine Lehre & $5,5(1-6)$ & \multirow{3}{*}{$\mathrm{p}=0,84$} \\
\hline & & 109 & Lehre & $5(3,5-7)$ & \\
\hline & & 61 & höherer Schulabschluss & $5(3,5-7)$ & \\
\hline & \multirow[t]{3}{*}{ Homöopathie } & 18 & keine Lehre & $3,5(1-6,25)$ & \multirow{3}{*}{$\mathrm{p}=0,50$} \\
\hline & & 106 & Lehre & $5(3-7)$ & \\
\hline & & 62 & höherer Schulabschluss & $4(2-6)$ & \\
\hline & \multirow[t]{3}{*}{ Kombination } & 17 & keine Lehre & $5(3-8)$ & \multirow{3}{*}{$\mathrm{p}=0,278$} \\
\hline & & 106 & Lehre & $7(5-8)$ & \\
\hline & & 60 & höherer Schulabschluss & $7(5-8)$ & \\
\hline
\end{tabular}

\section{Diskussion}

\section{Inanspruchnahme der Komplementärmedizin}

In dieser Studie wird der Wunsch nach Komplementärmedizin von 74,5\% der Patienten geäussert (Abschlussfrage 3). Sie würden gerne neben der Schulmedizin auch pflanzliche und/oder homöopathische Behandlungsmöglichkeiten angeboten bekommen. Diese Ergebnisse bestätigt die Studie von Härtel und Volger [6], die Männer und Frauen zwischen 19 und 69 Jahren nach ihrer Haltung zur Naturheilkunde befragt haben. Die Phytotherapie wurde gemäss den Autoren von 33\% der Frauen und 20\% der Männer und die Homöopathie von 11\% der Frauen und 6\% der Männer in Anspruch genommen. 58\% der Patienten würden es bevorzugen, wenn die Ärzte Komple- mentärmedizin öfters verschreiben und mehr Informationen dazu geben würden.

\section{Ähnlicher Stellenwert der Phytotherapie und Homöopathie bei den Patienten}

Das überraschendste Resultat dieser Studie stellt die beinahe gleichwertige Stellung der Wirksamkeit und des Nebenwirkungsprofils von pflanzlichen und homöopathischen Medikamenten bei den Befragten dar. Die eigenständige Homöopathie scheint der Phytotherapie in der Gunst der Patienten in nichts nachzustehen. Es könnte sein, dass das günstige Nebenwirkungsprofil beider Medizinrichtungen eine ähnliche Wirksamkeit suggeriert. Eine andere Interpretation führt zu der Überlegung, 
dass den Patienten, trotz vorheriger kurzer Aufklärung, der Unterschied zwischen Homöopathie und Phytotherapie zu wenig bewusst war. Dies zeigt sich auch im Praxisalltag, in dem die Patienten immer wieder über die verschiedenen Arten der Komplementärmedizin aufgeklärt werden müssen. Es bedarf deshalb weiterer Bemühungen, um die wissenschaftlich nachgewiesene Wirksamkeit der Komplementärmedizin sowohl in der Ärzteschaft als auch bei den Patienten bekannter zu machen. In einer 2009 durchgeführten Studie zeigten Deglon et al. [7], dass nur 14,2\% der Grundversorger einen Fähigkeitsausweis der Komplementärmedizin hatten (TCM/Akupunktur, Homöopathie, Neuraltherapie, Anthroposophische Medizin und/oder Phytotherapie). In einer anderen Studie, die im Akutspital Wattwil durchgeführt wurde, zeigten die Ärzte Unsicherheit und Skepsis, die Phytotherapie im Akutspital anzuwenden, während phytotherapeutische Anwendungen bei den Patienten auf eine hohe Akzeptanz stiessen [8]. Um dem Wunsch der Patienten besser nachkommen zu können, wäre der Aufbau eines fundierten ärztlichen Wissens wichtig. Die Gesellschaften der Union schweizerischer komplementärmedizinischer Ärzteorganisationen bieten die vier relevanten Fachrichtungen (Phytotherapie, Homöopathie, Anthroprosophische Medizin und TCM) an. Für die Phytotherapie engagiert sich in der Schweiz die Schweizerische Medizinische Gesellschaft für Phytotherapie (SMGP).

\section{Behandlung bei Depression}

Gemäss der Umfrage bevorzugen die Patienten, insbesondere die bereits psychisch Erkrankten, bei Depression die Schulmedizin. Dies überrascht, da mittlerweile Johanniskraut in der Behandlung der leichten bis mittelschweren Depression den synthetischen Antidepressiva gleichgestellt ist. Die gleiche Wirksamkeit bei besserer Verträglichkeit konnten Linde et al. [9] in einer Metaanalyse von 2008 bestätigen. Darin untersuchten die Autoren 29 Studien mit insgesamt 5489 Patienten - davon 19 Vergleichsstudien mit Placebo und 17 Vergleichsstudien mit synthetischen Antidepressiva. In einer anderen Studie verglich Schrader [10] Johanniskraut direkt mit Fluoxetin. Dabei fand er zwar eine leicht höhere ResponderRate unter Fluoxetin, jedoch bei einem deutlich besseren Nebenwirkungsprofil von Johanniskraut. Die gute Wirksamkeit des Johanniskrauts bzw. dessen Gleichstellung mit synthetischen Antidepressiva bei gutem Wirksamkeitsprofil scheint aber zu wenig in der Bevölkerung bekannt zu sein. Vermutlich bestehen bei den Patienten Vorurteile gegenüber den verschiedenen Behandlungsarten. Im Praxisalltag zeigt sich erfahrungsgemäss, dass einerseits die Wirkung der Komplementärmedizin oft unterschätzt und andererseits die Nebenwirkungen der synthetisch hergestellten Arzneimittel eher überschätzt werden. Wie bereits erwähnt, setzen in dieser Studie viele Patienten die Schulmedizin bei der Behandlung der Depression an die erste Stelle. Diese Resultate stehen im Gegensatz zu einer Untersuchung des BAG aus dem Jahr 2004, in der eine grössere Anzahl von Patienten eine komplementärmedizinische Behandlung bei Depression in Anspruch genommen hat [4]. Dieser Unterschied ergibt sich möglicherweise daraus, dass Johanniskrautpräparate in Apotheken ohne Rezept erhältlich sind und die Patienten solche Präparate bereits angewendet haben, bevor sie synthetisch hergestellte Medikamente und auch die entsprechende psychotherapeutische Behandlung ärztlicherseits in Anspruch genommen haben. Gemäss Saller (in Linde et al. [9]) besteht bei pflanzlichen Wirkstoffen eine Dosis-Wirkungs-Beziehung, die jedoch nur wenig erforscht ist. Für Johanniskrautextrakte liegen klinische Studien vor, die eine dosisabhängige antidepressive Wirksamkeit zeigen. Auch das klinische Interaktionspotenzial ist dosisabhängig, allerdings bezogen auf den Wirkstoff Hyperforin.

Bei Extrakten aus Vitex agnus-castus und Cimicifuga, die zur Behandlung des prämenstruellen Syndroms bzw. in den Wechseljahren eingesetzt werden, zeigte sich eine dosisabhängige Zunahme der Wirksamkeit bei gleichbleibender Häufigkeit unerwünschter Wirkungen. Die Ursache der eher niedrigen Dosierungsempfehlungen sehen Meier et al. [11] aufgrund einer direkten Übernahme aus einschlägigen Drogenmonographien. Möglicherweise sind die Dosisempfehlungen für viele pflanzliche Zubereitungen zu niedrig angesetzt, weil sie dadurch eher als «sicher» eingestuft werden, was wiederum zu einer Unterschätzung des Potenzials einer Reihe von Phytotherapeutika führen kann [11]. Aufgrund der nicht ausreichenden Wirksamkeit wird dann von einem Arzt in einer schulmedizinisch tätigen Praxis auch eine entsprechende «schulmedizinische» Medikation erwartet.

\section{Behandlung bei Magen-Darm-Beschwerden}

Bei Magen-Darm-Erkrankungen besteht eine lange Tradition, pflanzliche Heilmittel anzuwenden. Daher ist es nachvollziehbar, dass die Patienten primär die Phytotherapie vorziehen, jedoch eine höhere Wirksamkeit von der Schulmedizin erwarten. Erst in den letzten Jahren konnte eine Wirksamkeit von Phytotherapeutika (Iberogast, Pfefferminzöl) in Studien nachgewiesen werden. Khanna et al. [12] fanden 5 von 9 Studien mit Pfefferminzöl bei einem unruhigen Magen-Darm-Trakt, die eine Überlegenheit gegenüber Placebo zeigten. Für das Arzneimittel Iberogast wurde die Wirksamkeit in mehreren randomisierten, klinischen Doppelblindstudien belegt 
[13]. Auch hier bedarf es weiterer Aufklärungsarbeit, um das Vertrauen in die Phytotherapie zu stärken.

\section{Altersabhängige Daten}

Die jüngeren Patienten bevorzugen eher die Phytotherapie und Homöopathie gegenüber schulmedizinischen, medikamentösen Behandlungen. Diese Aussage deckt sich mit einer Studie von Stjernberg et al. [14] aus dem Jahr 2006. Diese kam zu dem Ergebnis, dass die Patienten umso weniger Phytotherapie nutzen, je älter sie sind. Es wurden in dieser Studie aber nur Altersgruppen über 60 Jahre untersucht [14]. Die Gründe dafür, warum die jungen Generationen die Phytotherapie mehr nutzen als die älteren, sind uns nicht bekannt.

\section{Geschlechterspezifische Unterschiede}

In der Studie des BAG von 2004 wurde, wie in unserer Studie, die Komplementärmedizin in höherem Masse von Frauen genutzt [4]. Im Vergleich waren von 1715 komplementärmedizinischen Benutzern 1197 Frauen und nur 518 Männer.

Warum Frauen eher als Männer komplementärmedizinische Arzneimittel bevorzugen, bleibt auch nach dieser Studie unklar. Es konnte nur eine Studie gefunden werden, die Eigenschaften von Personen untersuchte, welche komplementärmedizinische Methoden in Anspruch genommen haben. Dabei wurden Studierende, die sich komplementärmedizinisch bzw. schulmedizinisch behandeln liessen, miteinander verglichen. Es wurde festgestellt, dass Studenten, die ausschliesslich eine komplementärmedizinische Behandlung in Anspruch nahmen, eher davon überzeugt sind, ihre Gesundheit selbst kontrollieren zu können.

\section{Einfluss der Schulbildung}

Der Einfluss der Schulbildung auf die bevorzugte Therapieart ist in dieser Studie nur gering. Es zeichnet sich allenfalls eine Tendenz ab, dass Patienten mit einer zusätzlichen Ausbildung zur obligatorischen Schulzeit die Wirksamkeit der Phytotherapie/Homöopathie bei nicht lebensbedrohlichen Erkrankungen höher erachten als $\mathrm{Pa}-$ tienten ohne Lehre. In der Studie des BAG zeigt sich auch ein geringer Unterschied mit einer Wahrscheinlichkeit von 11,1\%, dass Personen mit höherer Schulbildung auf eine komplementärmedizinische Behandlung zurückgreifen - im Vergleich zu 8,5\% mit obligatorischem Schulabschluss [4]. In dieser Hinsicht hat sich in den letzten 10 Jahren somit nicht viel verändert.

\section{Stärken und Schwächen der Studie}

In unserer Studie wurde die Behandlung von häufigen und bekannten Erkrankungen thematisiert. Die Patienten wussten somit relativ gut, worüber sie zu urteilen haben. Hingegen wurden die Erkrankungen weder näher definiert noch präzise beschrieben, um nicht belehrend $\mathrm{zu}$ wirken. Insbesondere beim «Herzinfarkt» wurde nicht klar definiert, ob es sich um eine Primärprophylaxe, eine Sekundärprophylaxe oder gar um eine Akutbehandlung handelt. Die Beurteilung der Erkrankungsart und -schwere wurde den Teilnehmern überlassen. Diese schätzten den Herzinfarkt sicher als schwerwiegende Erkrankung ein und bevorzugten daher auch die Schulmedizin. Eine Vertiefung der Fragestellung in Primär- und Sekundärprophylaxe würde sicher auch einen interessanten Ansatz darstellen.

Irrtümlicherweise wurde bei der Frage nach der Wirksamkeit beim Herzinfarkt das Wort «stark» ausgelassen. Alle anderen Fragen wurden einheitlich formuliert. $\mathrm{Ob}$ dieser Umstand die Beurteilung der Patienten beeinflusst hat, ist nicht bekannt. Der Effekt dürfte jedoch gering sein, da die Antwortmöglichkeit auf einer Ordinalskala von 1-10 einheitlich war und in Bezug auf die Fragestellung erklärt wurde.

In der Studie des BAG von 2004 wurde auch untersucht, wie viele Personen sich in den letzten 12 Monaten in ärztliche Behandlung begeben haben und wie viele davon eine komplementärmedizinische Methode gewählt haben [4]. Dabei haben nur 5,4\% eine komplementärmedizinische Leistung wegen Herzbeschwerden in Anspruch genommen, was wiederum mit der Annahme der Patienten in unserer Studie übereinstimmt, dass die schulmedizinischen Medikamente besser wirksam sind.

Es wurde ein möglichst unselektioniertes Patientengut befragt. Die Demographie der Studienpopulation entspricht im Wesentlichen dem Patientenkollektiv der teilnehmenden Praxen. Somit erscheint eine systematische Verzerrung unwahrscheinlich, und die externe Validität könnte gross sein. Die «Responder-Rate» von 0,6 genügt wissenschaftlichen Standards. Laut Angaben der Patienten waren die Fragen verständlich formuliert. Bedingt durch die verhältnismässig kurze Einschlussperiode war die Patientenzahl relativ gering. Die Infrastruktur und der teilweise hektische Tagesablauf einer Arztpraxis erlaubten kein längerfristig angelegtes Studienprotokoll. Deshalb ist diese Studie als «Stichprobenanalyse» zu betrachten. Einige signifikante Studienergebnisse könnten durch multiples Testen zufällig zustande gekommen sein. Zudem stellt sich manchmal die Frage nach der klinischen Relevanz eines Resultats. Weil eine möglichst detailliert abgestufte Antwortskala von 1-10 benutzt wurde, konnten geringe Unterschiede erfasst werden. Geringe Unterschiede könnten dann einerseits die Relevanz der Resultate infra- 
ge stellen und andererseits auch die Unsicherheit, die Unschlüssigkeit oder das Unwissen der Probanden widerspiegeln. Wäre ein grobes Skalenniveau gewählt worden, hätte man eventuell fälschlicherweise relevante Unterschiede suggeriert. Der Vorteil einer fein abgestuften Ordinalskala von 1-10 bietet die Möglichkeit der Darstellung von signifikanten Tendenzen. Es wurde schliesslich nur nach der «Meinung» der Patienten gefragt.

Basierend auf den erhaltenen Resultaten sollten die Patienten vermehrt darauf aufmerksam gemacht werden, dass die wissenschaftliche Evidenz in der Phytotherapie während der letzten Jahre grosse Fortschritte gemacht hat. Natürlich kann aufgrund der relativ kleinen Patientenzahl nur von einer «Stichprobenanalyse» gesprochen werden. Zudem kann man davon ausgehen, dass sich in schulmedizinischen Praxen ein Patientengut findet, das ausschliesslich auf die Wirksamkeit der Schulmedizin setzt. Dennoch wird die Wirksamkeit einer Kombination von schulmedizinischen und komplementärmedizinischen Medikamenten mit einem Median von $>5$ als hoch eingeschätzt. Interessant wäre es zu wissen, zu welchen Resultaten eine identisch gestaltete Studie in Praxen mit komplementärmedizinischem Schwerpunkt führt.

\section{Schlussfolgerung}

Die Patienten beurteilen die Phytotherapie und Homöopathie sehr ähnlich. Seit der Studie des BAG hat sich die Patienteneinstellung trotz neuen wissenschaftlichen
Erkenntnissen nicht grundsätzlich verändert. Gemäss unserer Studie ziehen die Hausarztpatienten bei MagenDarm-Erkrankungen eine phytotherapeutische Behandlung vor, obwohl sie die Wirksamkeit der Schulmedizin höher einstufen. Zur Behandlung der Depression wird aber die Schulmedizin der Komplementärmedizin vorgezogen. Die positiven Resultate der wissenschaftlichen Forschung in der Komplementärmedizin, insbesondere in der phytotherapeutischen Behandlung der Depression, sollten an die Patienten weitergegeben werden. Dies ist wiederum eine ärztliche Aufgabe. 74\% der Patienten, insbesondere Frauen, jüngere Patienten sowie Patienten mit weiterführender Ausbildung nach der obligatorischen Schulzeit, schätzen das Angebot an komplementärmedizinischen Massnahmen - insbesondere Phytotherapie oder Kombinationstherapien. Diesen Patientengruppen sollte die Komplementärmedizin nicht vorenthalten werden.

\section{Dank}

Dank gilt den Kollegiumsmitgliedern Georg Stoffel und Daniel Reuss für die Hilfe bei der Patientenrekrutierung und der Datenerhebung sowie PD Dr. med. Jens Hellermann für die Durchsicht der statistischen Daten.

\section{Disclosure Statement}

Die Autoren geben an, dass keine Interessenkonflikte bestehen. Diese Studie wurde von Silke Henzen als Abschlussarbeit zur Erlangung des Fähigkeitsausweises Phytotherapie (SMGP) durchgeführt.

\section{Literatur}

1 Nahin RI, Barnes PM, Stussman BJ, Bloom B Cost of complementary and alternative medicine (CAM) and frequency of visits to CAM practitioners: United States, 2007. Natl Health Stat Report 2009;18:1-14. https://nccih.nih. gov/sites/nccam.nih.gov/files/news/camstats/ costs/nhsrn18.pdf.

2 Institut für Demoskopie Allensbach: Naturheilmittel 2010. Ergebnisse einer bevölkerungsrepräsentativen Befragung. Allensbach am Bodensee, Institut für Demoskopie Allensbach, 2010.www.ifd-allensbach.de/uploads/ tx_studies/7528_Naturheilmittel_2010.pdf.

3 Amt für Statistik Fürstentum Liechtenstein Liechtensteinische Gesundheitsbefragung 2012. Vaduz, Amt für Statistik, 2012. www.llv. li/files/as/Gesundheitsbefragung_2012.pdf.

4 Bundesamt für Gesundheit (BAG): Inanspruchnahme von 5 Therapien der Komplementärmedizin in der Schweiz. Statistische Auswertung auf der Basis der Schweizerischen Gesundheitsbefragung 1997 und 2002 September 2004. www.bag.admin.ch/themen/ krankenversicherung/00263/00264/04102/ index.html?lang=de.
5 Sibalic V, Henzen S, Stoffel G, Reuss D, Stoller G, Scheiwiler P: The young generation of patients seems to favor complementary medicine in Switzerland. The HoPla-Study. Swiss Family Docs Kongressposter, Abstractpublikation. Primary Care 2014; 14:230.

$\checkmark 6$ Härtel U, Volger E: Inanspruchnahme und Akzeptanz klassischer Naturheilverfahren und alternativer Heilmethoden in Deutschland - Ergebnisse einer repräsentativen $\mathrm{Be}$ völkerungsstudie. Forsch Komplementarmed Klass Naturheilkd 2004;11:327-334.

7 Deglon-Fischer A, Barth J, Ausfeld-Hafter B: Komplementärmedizin in Schweizer Praxen der Grundversorgung. Forsch Komplementmed 2009; 16:251-255.

8 Wemmer D: Pilotprojekt zur Integration von Phytotherapie in der Akutmedizin. Schweiz Z Ganzheitsmed 2012;24:279-283.

$>9$ Linde K, Berner MM, Kriston L: St. John's wort for major depression. Cochrane Database Syst Rev 2008;4:CD000448.

10 Schrader E: Equivalence of St. John's wort extract (Ze 117) and fluoxetine: a randomized, controlled study in mild-moderate depression. Int Clin Psychopharmacol 2000;15:61-68.
11 Meier B, Knöss W, Wenng A, Saller R: Blick in die Zukunft pflanzlicher Arzneimittel. Forsch Komplementmed 2014;21(suppl 1):2934.

12 Khanna R, MacDonald JK, Levesque BG: Peppermint oil for the treatment of irritable bowel syndrome: a systematic review and meta-analysis. J Clin Gastroenterol 2014;48: 505-512.

13 Allescher H-D, Wagner H: STW 5/Iberogast: Multi-Target-Wirkung bei funktioneller Dyspepsie und Reizdarmsyndrom. Wien Med Wochenschr 2007;157:301-307.

14 Stjernberg L, Berglund J, Halling A: Age and gender effect on the use of herbal medicine products and food supplements among the elderly. Scand J Prim Health Care 2006;24: 50-55. 